V.

\title{
A Case of Subcutaneous Emphysema During Labour.
}

\author{
By Robert Jardine, M.D., etc., \\ Professor of Midwifery, St. Mungo's College, Glasgow; Senior \\ Physician, Glasgow Maternity Hospital, etc.
}

Mrs. K., æt 22, at full time of her first pregnancy, was admitted to the Glasgow Maternity Hospital on the afternoon of February 10th, 1907. A midwife had been in attendance and had ruptured the membranes about 1 a.m. on the 10th. The patient had vomited a great deal, and about 5 a.m. on the 10th she had experienced a choking sensation and fulness in her neck. With each attack of vomiting there was an increase of the swelling and it quickly spread to the face and eye-lids. A doctor was called in, and he at once sent the patient into Hospital.

On admission, the patient was in a very exhausted condition. Her face was much swollen and very odematous looking - the facies of acute nephritis-but on palpation the face, neck, chest, and a portion of the abdominal wall were found to be extensively emphysematous, crepitations being easily felt. The condition was more marked on the right side. There was very little discomfort except on deep inspiration, when the patient complained of lancinating pain over the apices of both lungs. The pulse was very feeble, 130 . The retraction ring was present, and the bladder much distended. The os was the size of a crown piece, and the head presented, but was not engaged. The diagonal conjugate measured $3 \frac{1}{2}$ in.

The os was dilated manually and the forceps was applied but not persevered with, as there was little hope of delivering therewith. Craniotomy was performed and delivery effected by means of the combined instrument. During delivery the patient was in a very critical condition. At times, her breathing was very shallow and her pulse so feeble and rapid that it could not be counted. Strychnine $1 / 30 \mathrm{gr}$. was given hypodermically, and also $20 \mathrm{~m}$. extract of ergot. The strychnine was continued in $1 / 60 \mathrm{gr}$. doses four-hourly for a few days.

The emphysema gradually disappeared and was gone on the seventh day after delivery. The recovery was uninterrupted, al- 
though we fully expected the patient would become septic, as the midwife was reported to have examined very frequently.

A careful examination of the chest revealed nothing except that the breath sounds over the apex of the right lung were somewhat harsh, but there were no râles. There was a slight cough but no expectoration.

The usual explanation of these cases of subcutaneous emphysema occurring during labour is that the air comes from a ruptured vesicle in the apex of the lungs, but Herrgott maintains that it is the trachea or bronchus which ruptures during expiration associated with a violent effort or cry. In this case the lancinating pain complained of at the apices of the lungs during deep inspiration would seem to point to the rupture having occurred in that region.

Subcutaneous emphysema is a very rare complication of labour. This is the only case $I$ have ever seen. There is practically no danger from it, but when it occurs the labour should be finished as soon as possible. 